\author{
Marquette University \\ e-Publications@Marquette
}

Biomedical Engineering Faculty Research and

Publications

Biomedical Engineering, Department of

$1-2009$

\title{
Functional Stability of Transfemoral Amputee Gait Using the 3R80 and Total Knee 2000 Prosthetic Knee Units
}

M. Barbara Silver-Thorn

Marquette University, barbara.silver-thorn@marquette.edu

Christine L. Glaister

Marquette University

Follow this and additional works at: https://epublications.marquette.edu/bioengin_fac

Part of the Biomedical Engineering and Bioengineering Commons

\section{Recommended Citation}

Silver-Thorn, M. Barbara and Glaister, Christine L., "Functional Stability of Transfemoral Amputee Gait Using the 3R80 and Total Knee 2000 Prosthetic Knee Units" (2009). Biomedical Engineering Faculty Research and Publications. 348.

https://epublications.marquette.edu/bioengin_fac/348 
Marquette University

e-Publications@Marquette

\title{
Biomedical Engineering Faculty Research and Publications/College of
} Engineering

This paper is NOT THE PUBLISHED VERSION; but the author's final, peer-reviewed manuscript. The published version may be accessed by following the link in the citation below.

JPO Journal of Prosthetics and Orthotics, Vol. 21, No. 1 (January 2009): 18-31. DOI. This article is (C) Wolters Kluwer and permission has been granted for this version to appear in $\underline{\mathrm{e}-}$

Publications@Marquette. Wolters Kluwer does not grant permission for this article to be further copied/distributed or hosted elsewhere without the express permission from Wolters Kluwer.

\section{Functional Stability of Transfemoral Amputee Gait Using the 3R80 and Total Knee 2000 Prosthetic Knee Units}

\author{
M. Barbara Silver-Thorn \\ Department of Biomedical Engineering, Marquette University, Milwaukee, Wisconsin \\ Christine L. Glaister \\ Department of Biomedical Engineering, Marquette University, Milwaukee, Wisconsin
}

Disclosure: The authors declare no conflict of interest.

This work was supported by the Department of Biomedical Engineering at Marquette University and the Department of Physical Medicine and Rehabilitation at the Medical College of Wisconsin.

\footnotetext{
Abstract in Brief

Prosthetic knee designs have become extremely sophisticated compared to early constant friction single axis mechanisms. Today, prosthetists and physicians have a wide variety of components to choose from for their transfemoral amputee patients. Unfortunately, there are little quantitative data comparing the performance of
} 
different types of prosthetic components, and prosthetic prescription is often based on empirical knowledge and experience, rather than objective comparison of prosthetic designs. This study used gait analysis to objectively evaluate the stability characteristics of two types of prosthetic knee designs, the polycentric Total Knee 2000 (Ossur, Aliso Viejo, CA) and the single axis 3R80 stance control knee (Otto Bock Healthcare, Minneapolis, MN). Five healthy, active transfemoral amputees participated in this study by completing two gait analysis sessions, one with the Total Knee 2000 and one with the 3R80. At the end of each session, subjects were asked to complete a questionnaire for comparison of their subjective preferences with the objective biomechanical measures from the gait analysis. Kinematic and kinetic gait data were collected. Stride and temporal parameters (i.e., velocity, cadence, stride length, step length, stance and swing phase durations, and single and double support durations), joint angles and moments were computed from the Vicon data. A within subjects statistical analysis was then conducted, using repeated measures ANOVA, to determine if there were significant differences in performance between the two knee designs. The stride and temporal parameters computed in this study indicated that the Total Knee 2000 demonstrated a high degree of overall stability compared to the 3R80. The differences in inherent mechanical stability in the Total Knee 2000 and the $3 R 80$ resulted in different calculated hip moments. The corresponding required hip moments indicated that the relative stability of the two knee designs changed throughout the gait cycle due to the polycentric nature of the Total Knee 2000. Specifically, the Total Knee 2000 was found to be less stable than the $3 R 80$ in early stance and more stable than the 3R80 in mid- and late-stance. In addition to the biomechanical evidence of increased stability with the Total Knee 2000, the responses to the subjective questionnaires indicated that most subjects felt more stable and more confident bearing weight on the Total Knee 2000 than the 3R80.

Transfemoral (TF) amputees must overcome the loss of two major joints (the ankle and knee) and the loss or partial loss of many of the lower limb muscle groups involved in ambulation. Although prosthetic components may replace some of the functionality of the amputated limb, they cannot replicate the activity of lost musculature. As a result, there are some distinct differences in ambulation between able-bodied subjects and TF amputees.

Many of the differences in stride and temporal parameters exhibited by TF amputees in comparison with nonamputees may be associated with the stability of the prosthetic limb. Decreased stability on the prosthetic limb compared with the sound limb may lead to the decreased prosthetic single support durations seen in TF amputees. This decrease in prosthetic single support duration reduces the time available for advancing the sound limb, leading to shorter sound side steps, shorter strides, and slower velocities. ${ }^{1-4}$

Although TF amputees cannot generate a large hip extension moment on the prosthetic side due to the loss of the amputated muscle tissue, this mechanism is important for prosthetic stability. A hip extension moment in early stance is commonly used by TF amputees to provide voluntary stability of the prosthetic knee..$^{5-8}$ Similarly, a large hip flexion moment seen on the prosthetic side is a typical mechanism used to overcome the stability of the prosthetic knee component and initiate knee flexion for swing phase. ${ }^{8,9}$

Prosthetic knees must replicate the function of the human knee to provide stability for weight bearing in stance phase and controlled motion during swing phase. Instability in a prosthetic knee often leads to the adoption of gait deviations, increased energy cost for ambulation and may result in prosthetic knee buckling during stance. Improperly adjusted swing phase mechanics can also result in the adoption of gait deviations and an increase in the energy cost of ambulation. Prosthetists and physicians must consider each patient's abilities and functional goals to determine which prosthetic knee is most appropriate, and which will likely yield the smoothest, most reliable gait for each individual. ${ }^{10,11}$

During stance phase, the knee must provide stability for weight bearing while also facilitating the transition from stance to swing phase. The normal anatomical knee can fulfill these differing demands efficiently through the 
interaction of anatomical structures, muscle activity and the ground reaction force vector (GRFV). Standard prosthetic knee mechanisms do not replace the muscular components of the anatomical knee, and cannot adjust to these demands. As a result, there may be a trade-off for TF amputees between stance phase stability and the hip moment required to initiate swing phase, and this trade-off may result in the adoption of gait deviations and increased demand on the residual limb hip musculature.

Stance phase stability of a prosthetic knee design has two contributing factors: the inherent mechanical stability of the mechanism itself, and the voluntary stability provided by the remaining hip musculature of the TF amputee. Mechanical stability of a prosthetic knee is achieved by aligning the center of rotation of the prosthetic knee posterior to the GRFV throughout stance. This results in a passive extension moment at the knee, preventing knee buckling under weight bearing as long as the GRFV remains anterior to the knee axis of rotation. Voluntary or active stability of a prosthetic knee is achieved by the TF amputee activating the hip extensors to exert an extension moment at the hip from heel contact through mid-stance. This active extension moment at the hip produces an extension moment at the knee, preventing collapse of the knee under weight bearing. ${ }^{12-14}$ From mid- through terminal stance, the GRFV is once again located anterior to the knee joint, and knee extension is passively maintained through alignment stability.

Providing stability during stance is not the only function of prosthetic knees. A smooth transition from stance to swing phase and controlled motion of the knee throughout swing phase are equally important aspects of normal gait. Controlled motion of the knee during swing phase can be provided through the use of swing phase control mechanisms such as constant friction and hydraulic devices. The transition between the two phases of gait, however, is a more difficult function to replicate. Initiation of swing phase requires flexion of the knee joint. This entails deactivating any stance phase control mechanisms and overcoming the alignment stability of the prosthetic knee. Deactivation of stance phase control usually occurs as the GRFV moves anterior to the knee joint in mid- to late-stance. Overcoming the alignment stability of the prosthetic knee, however, requires that the amputee actively exert a flexion moment at the hip. This moves the GRFV posterior to the center of rotation of the prosthetic knee and allows the amputee to flex the knee and initiate swing. Prosthetic knee designs that provide increased alignment stability during stance phase may require an increased hip flexion moment to initiate knee flexion for swing phase, resulting in a trade-off between stance phase stability and swing phase initiation effort. ${ }^{9,12,15,16}$

The Total Knee 2000 and the 3 R80 prosthetic knees are both indicated for TF amputees who lead moderately active lives, are able to vary their cadence, and have good voluntary control of their prostheses. ${ }^{17,18}$ Both knees are equipped with stance phase control to provide stance phase stability; however, these two components achieve this goal through very different mechanisms. The Total Knee 2000 is a polycentric prosthetic knee with a geometric locking system to provide stance phase stability, while the $3 R 80$ is a single axis prosthetic knee that utilizes a hydraulic cylinder to provide stance phase stability. The inherent mechanical differences between the single axis and polycentric prosthetic knee mechanisms and the two types of stance phase control mechanisms in the Total Knee 2000 and the $3 R 80$ result in very different mechanical stability characteristics.

A number of biomechanical measures have been used to compare both the overall stability of amputee gait and the stability of prosthetic knee designs. Stride length, step length, velocity, cadence and the durations of stance and swing phases have all been suggested as indicators of overall prosthetic stability. Changes in sound side step length and stride length between prostheses may be influenced by the overall stability of the prosthesis. Increased stability of the prosthesis should theoretically allow the amputee to maintain prolonged single limb support over the prosthesis which, in turn, would result in longer steps with the sound limb. ${ }^{1,4}$ Cadence and velocity are both related to stride length and step length (i.e., increased step length with a constant cadence will result in increased velocity), and have also been suggested as indicators of overall prosthetic performance. 
Velocity in particular has been correlated with the degree of lower limb impairment, ${ }^{19}$ with decreased velocity an indicator of increased impairment.

The duration of the stance and swing phases of gait have also been identified as indicators of overall prosthetic stability by a number of investigators. ${ }^{2,3,20-22}$ Lower limb amputees typically spend more time in sound limb stance than in prosthetic limb stance. Comparing two prostheses, a more stable prosthesis would be expected to result in more symmetric stance and swing phase durations, because amputees should feel more secure in single limb stance on a more stable prosthesis.

The hip extension and flexion moments on the prosthetic side during gait are also likely to be closely related to the stability of a prosthetic knee. If the knee joint is mechanically stable, the amputee need not utilize their residual limb hip extensors to maintain voluntary stability at the knee. However, if the knee joint is not mechanically stable, the amputee must use their residual limb hip extensors and exert an extension moment at the hip to maintain voluntary stability of the knee. ${ }^{10,12,13}$ In comparing two prostheses in terms of hip moment, a prosthesis that is more stable should result in a decreased hip extension moment during stance phase, indicating that the prosthesis has greater inherent mechanical stability and less need for the amputee to use their residual limb hip extensors to achieve voluntary stability. In addition, swing phase initiation requires flexion of the knee which is often achieved by the amputee activating their hip flexors. A more stable prosthesis should require a larger hip flexion moment in preswing to overcome the stability of the prosthetic knee and initiate flexion for swing phase. Calculating the hip extension moment to maintain stability in stance phase and the hip flexion moment required to initiate swing phase can be used not only to compare the stability of two prosthetic knee designs, but can also potentially be used to determine whether there is a trade-off between stance phase stability and swing phase initiation effort. ${ }^{23,24}$

The current study considers two different knee designs with different stability mechanisms to determine whether the same trade-offs between stance stability and swing phase initiation effort, seen previously for alignment variations, are valid for different degrees of inherent knee component stability.

\section{METHODS}

Five healthy, active unilateral TF amputees were recruited through prosthetist referral to participate in this study (Table 1). Subjects were required to have a stable residual limb volume free from infection and inflammation, be $\mathrm{K} 2$ (ability or potential for ambulation with the ability to traverse low-level environmental barriers such as curbs, stairs, or uneven surfaces and is considered a typical community ambulator) or greater functional level ambulators, and be capable of repeated unassisted walking trials. Two subjects $(1,3)$ used a single axis knee with hydraulic stance phase control in their definitive prosthesis, two subjects $(2,5)$ used a constant friction polycentric knee with a geometric lock for stance phase control in their definitive prosthesis, and one subject (4) used a constant friction polycentric knee with an internal extension assist in his definitive prosthesis. All subjects used an energy storage and return foot in their definitive prosthesis.

Table 1: Subject characteristics and definitive prostheses

\begin{tabular}{|l|l|l|l|l|l|l|}
\hline Subject & $\mathbf{1}$ & $\mathbf{2}$ & $\mathbf{3}$ & $\mathbf{4}$ & $\mathbf{5}$ & $\begin{array}{l}\text { Mean } \\
\text { (std } \\
\text { dev) }\end{array}$ \\
\hline Age (yrs) & 33 & 43 & 49 & 41 & 58 & $\begin{array}{l}44.8 \\
(9.3)\end{array}$ \\
\hline $\begin{array}{l}\text { Time since } \\
\text { amputation } \\
\text { (yrs) }\end{array}$ & 8 & 20 & 31 & 34 & 2 & 19 \\
\hline
\end{tabular}




\begin{tabular}{|c|c|c|c|c|c|c|}
\hline $\begin{array}{l}\text { Cause of } \\
\text { amputation }\end{array}$ & Cancer & Trauma & Trauma & Trauma & Trauma & NA \\
\hline Weight (kg) & 84 & 86 & 115 & 98 & 87 & $\begin{array}{l}94 \\
(13.0)\end{array}$ \\
\hline Height $(\mathrm{cm})$ & 178 & 171 & 173 & 184 & 185 & $\begin{array}{l}178.2 \\
(6.3)\end{array}$ \\
\hline $\begin{array}{l}\text { Residual limb } \\
\text { length }(\mathrm{cm})\end{array}$ & 32 & 36 & 28 & 23 & 32 & $\begin{array}{l}30.2 \\
(5.0) \\
\end{array}$ \\
\hline $\begin{array}{l}\text { Current } \\
\text { prosthetic knee }\end{array}$ & C-Leg & TK1900 & $3 R 80$ & 3R36 & TK 2000 & NA \\
\hline $\begin{array}{l}\text { Current } \\
\text { prosthetic foot }\end{array}$ & $\begin{array}{l}\text { Otto Bock } \\
\text { Luxon } \\
\text { Journey foot }\end{array}$ & $\begin{array}{l}\text { College Park } \\
\text { Multi-axial } \\
\text { foot }\end{array}$ & $\begin{array}{l}\text { College Park } \\
\text { Multi-axial } \\
\text { foot }\end{array}$ & $\begin{array}{l}\text { Modular III } \\
\text { Flex Foot }\end{array}$ & $\begin{array}{l}\text { EndoliteMulti- } \\
\text { axial foot }\end{array}$ & NA \\
\hline
\end{tabular}

The study protocol was approved by the Institutional Review Boards of Marquette University and Froedtert Memorial Lutheran Hospital/Medical College of Wisconsin, and each subject gave their informed consent prior to initiation of research activities. The protocol for this comparative study was designed to isolate the prosthetic knee as the only controlled variable in the experimental prosthesis. As a result, each subject's experimental prosthesis replicated their definitive prosthesis as closely as possible, with the exception of the knee. All subjects used the prosthetic foot from their definitive prosthesis and alternately the $3 R 80$ single axis prosthetic knee with hydraulic stance phase control and the Total Knee 2000 with a geometric locking system to provide stance phase control in the experimental prosthesis. Three subjects used the socket from their definitive prosthesis in the experimental prosthesis, while duplicate sockets were fabricated for the remaining two subjects.

Each subject participated in an initial fitting appointment and a physical therapy (PT)/gait analysis session with the experimental prosthesis. Initial fittings took place at the prosthetists' offices, where temporary markings were made to allow replication of the prosthetic alignment at the gait analysis session. A final prosthetic fitting and PT session with the experimental prosthesis was conducted in the gait lab. During the final fitting and PT session, the prosthetist adjusted the resistance settings on the prosthetic knee to the "optimum" setting for each individual, while a licensed physical therapist used a gait belt to ensure the safety of the subjects while they adjusted to walking with the new prosthetic knee. The optimum setting for each subject was determined by: 1) adjusting the flexion resistance to prohibit excessive heel rise in late stance, and 2) adjusting the extension resistance to allow the shank to swing through smoothly in preparation for heel strike.

PT sessions continued for up to $1 \mathrm{hr}$, or until the prosthetist, physical therapist, and subject agreed that the subject was ambulating safely and consistently (defined as minimal variation in velocity during gait, e.g. standard deviation in velocity using the experimental prosthesis was less than or within $10 \%$ of the standard deviation for five timed walks with the subject's definitive prosthesis). At this point, the gait analysis session began. Reflective markers were applied to each subject with double sided adhesive tape on the anterior superior iliac spines, the sacrum (mid-way between the posterior superior iliac spines), and on the following locations on the sound limb: thigh, tibia, second metatarsal head, and calcaneus. The locations for the toe, ankle, and heel markers on the prosthetic side were estimated, as closely as possible, to reflect the corresponding sound side markers. The prosthetic shank marker was placed mid-way between the prosthetic knee and ankle markers, and the prosthetic thigh marker was placed mid-way between the prosthetic knee and the hip marker. A knee alignment device (Motion Lab Systems Inc., Baton Rouge, LA) was placed on the medial and lateral malleoli of the sound limb and on the axis of rotation of the prosthetic knee. This triad of markers was used while collecting static data to provide better definition of the knee axis of rotation than could be obtained with a single knee marker. After 
collecting static data, the knee alignment devices were replaced with single knee markers on the lateral malleolus of the sound limb and on the lateral side of the axis of rotation of the prosthetic knee.

Subjects were then asked to walk at a comfortable velocity along the data collection corridor while kinematic and kinetic data were collected. Kinematic data were collected at $120 \mathrm{~Hz}$ using a video-based Vicon 524 Motion Analysis System (Lake Forest, CA) with 15 cameras. Kinetic data were collected at $1800 \mathrm{~Hz}$ by two AMTI ORS6500 force plates (Watertown, MA). Kinetic and kinematic data were synchronized by the Vicon hardware. Walking trials continued until each subject made a total of five clean force plate strikes with each foot. A clean force plate strike occurred when one foot was placed completely inside the force plate area without the other foot coming into contact with the force plate. The subject's starting position was adjusted as necessary by the research staff to ensure clean force plate strikes; however, the subject was not informed of the location of the force plates to prevent planned foot placements which may deviate from normal gait patterns.

Data were processed with Vicon's full body kinetic and kinematic Golem model, ${ }^{25}$ with modifications made to account for the inertial properties of the prosthetic limb. ${ }^{26,27}$ Joint moments and angles were calculated using inverse dynamics and normalized to percent gait cycle. The individual time series (and calculated mean time series) for each clean walking trial for each subject were also calculated. The force plate data and subject measurements were also used to calculate the required hip moment for maintaining stability in stance phase, and the required hip moment for initiating swing phase. These calculations were based on Radcliffe's stability equation ${ }^{14,27}$ :

$$
M_{\mathrm{h}}=\left(\frac{L}{Y}\right)\left(P x-M_{\mathrm{k}}\right)
$$

where $M_{\mathrm{h}}$ is the required moment at the hip, $L$ is the distance from the hip to the heel, $Y$ is the distance from the knee center to the heel, $P$ is the vertical component of the GRFV, $x$ is the perpendicular distance between the GRFV and the knee center, and $M_{k}$ is the braking moment provided by the knee. The exact braking moments of the Total Knee 2000 and 3R80 throughout the gait cycle are not known (S. Elliott, Ossur, personal communication, 2005; K. Knox, Otto Bock, personal communication, 2005). The geometric lock on the Total Knee 2000 theoretically has no braking moment when the geometric lock is deactivated, and an infinite braking moment once the geometric lock is activated; there is no clear method, however, to identify the point at which the geometric lock becomes activated. The braking moment of the 3R80 in stance phase depends on the angular velocity at the knee and the resistance levels set by the prosthetist; no data for the braking moment supplied throughout stance phase is available. As a result, the knees were modeled as if they provided no braking moment, which effectively modeled the knees in a worst case, least stable scenario.

Radcliffe's stability equation gives a quasi-static estimate of the hip moment required to maintain stability in stance phase, and can also be used to estimate the hip moment required to initiate swing phase knee flexion. The slow speeds encountered during stance phase allow the use of this equation, despite the fact that it does not take into account inertial effects. Modeling the prosthetic knees with zero braking moments results in overestimation of the required hip extension moment for maintaining stability in stance phase once the stance control mechanisms are activated. To overcome this limitation, this study assumes that the stance control mechanisms are activated during loading response ( $0 \%$ to $10 \%$ of the gait cycle), and only the required hip moments in this portion of the gait cycle are considered. In this way, this equation can be used to make preliminary comparisons of the two prosthetic knee components investigated in this study. The required hip extension moment for maintaining stability in stance phase and the required hip flexion moment for initiating swing phase were calculated on the prosthetic side for each of the five trials and the time series were averaged together. 
After each gait analysis session, each subject was asked to complete a Borg's Rating of Perceived Exertion test and a short questionnaire concerning their perceptions of their stability. ${ }^{27}$ These tests were used to provide subjective data for comparison with the biomechanical data collected. The Borg's Rating of Perceived Exertion test asked subjects to circle the number (ranging from 6 to 20) that most closely corresponded to the level of exertion they felt while completing the walking trials; a rating of 6 on the Borg's scale corresponded to no exertion, while a rating of 20 corresponded to maximum exertion. The questions on the stability questionnaire asked the subjects to rate their confidence in weight bearing, their perceived stability and their comfort walking on uneven terrain, up stairs and in a crowd with each knee. These questions were scored on a numerical scale from 1 to 5 , with a rating of 1 corresponding to the least confidence in weight bearing, the lowest stability, and the least comfort on uneven terrain, walking up stairs and in a crowd. Note that subject 5 did not complete the subjective questionnaire after gait analysis with the 3R80. As a result, his responses were not included in the analysis of the subjective questionnaires.

The cross-over design of this study necessitated the use of a repeated measures ANOVA to determine whether there was a significant difference between the two knees. ${ }^{28}$ Values for the stride and temporal parameters were compared directly. The peak required hip extension moment during loading response and peak required hip flexion moment in terminal stance were identified from the time series for each of the five clean trials; these values were then used in the statistical analysis. Statistical tests were performed separately for each subject with the data from the two prosthetic knees.

\section{RESULTS}

Comparative temporal and stride parameters for each of the five subjects are shown in Figures 1-3. Velocity, cadence, stride length, and step length were computed by the Vicon software, based on the heel strike and toe off events that were manually identified. When multiple strides occurred in a single walking trial, the stride parameters for that trial were averaged over the number of complete strides; the mean values reported in Figures 1, 2 represent the average of the left and right sides for the five walking trials. Step length was examined in a similar manner for the prosthetic (Fig. 3A) and sound (Fig. 3B) sides and for each subject. Prosthetic stance and swing phase durations, as well as single and double support durations for both the prosthetic and sound limbs, are summarized for each of the five subjects in Figures 4, 5, respectively. Stance and swing phase durations are reported as a percentage of the gait cycle, while single and double support durations are reported as a percentage of the stride time. 

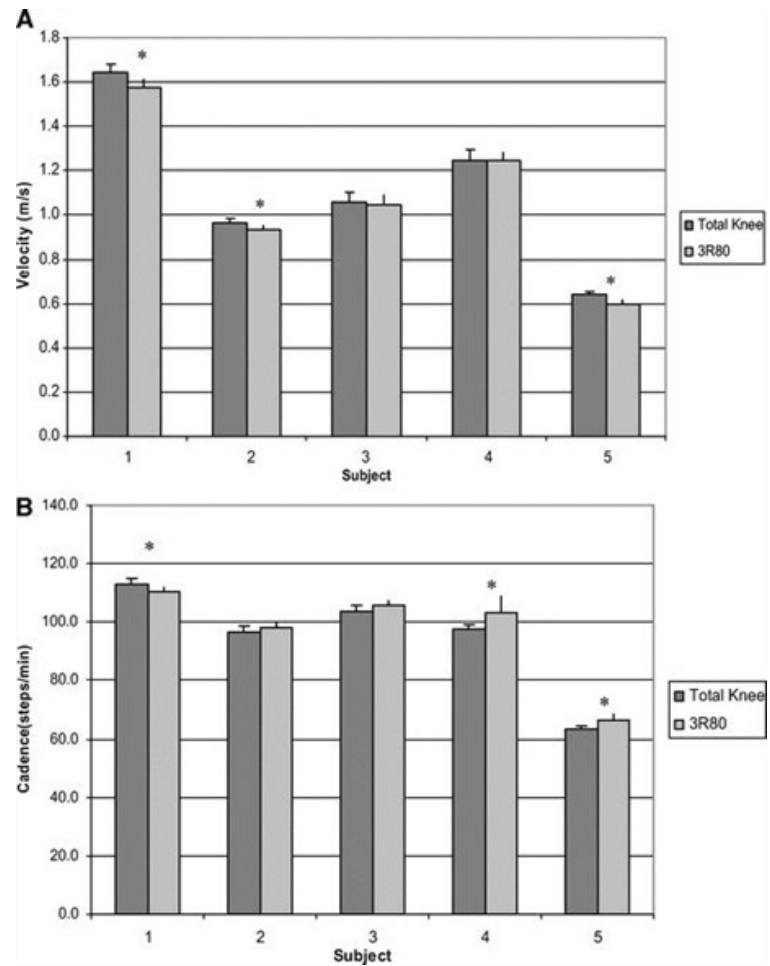

Figure 1.: Comparison of mean velocity (A) and cadence (B) for all subjects when ambulating with both the Total Knee and the 3R80. The asterisk reflects a statistically significant difference between values for the respective subject.

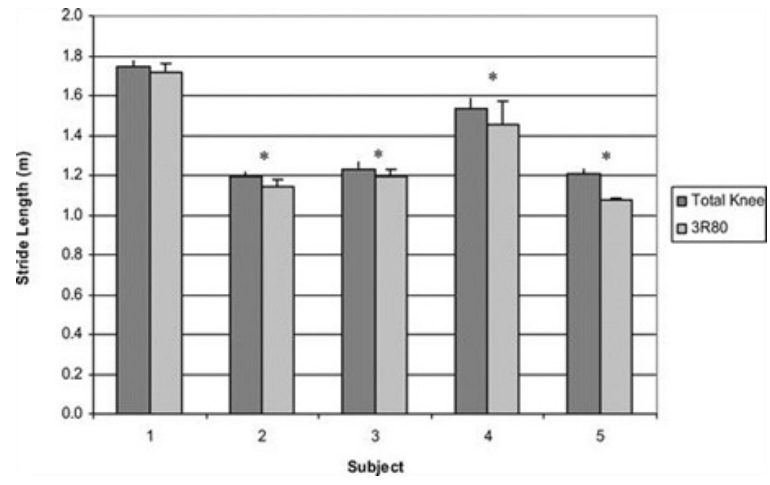

Figure 2.: Comparison of mean stride length for all subjects when ambulating with both the Total Knee and the 3R80. The asterisk reflects a statistically significant difference between values for the respective subject. 

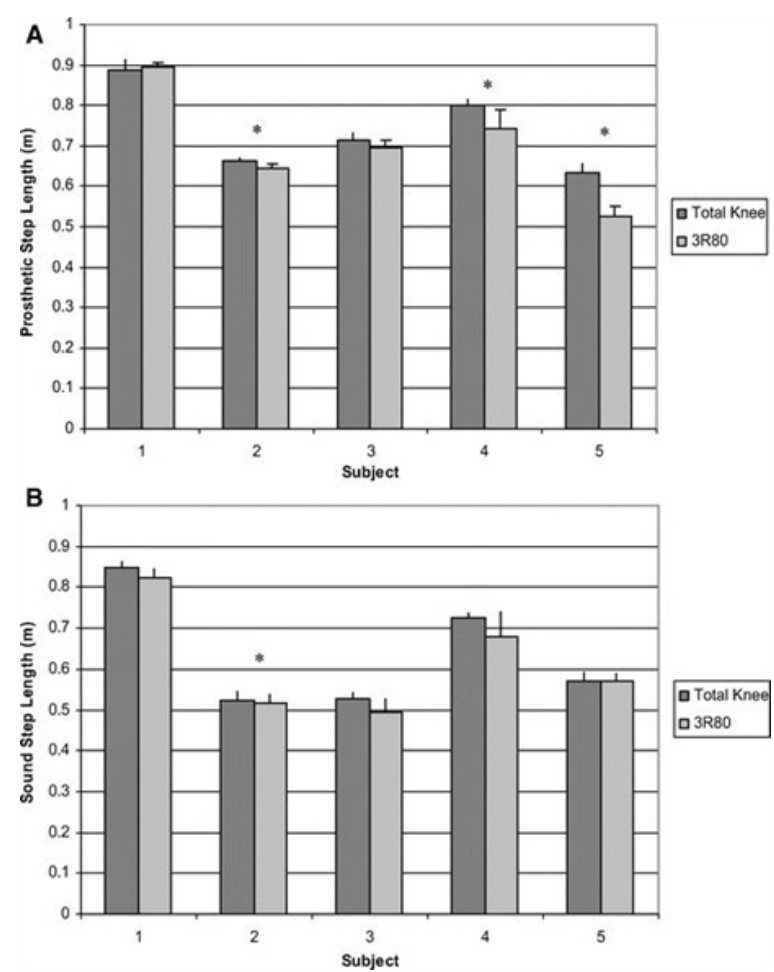

Figure 3.: Comparison of mean step \{lsqb;(A): prosthetic limb, (B): sound limb\{rsqb; for all subjects when ambulating with both the Total Knee and the 3R80. The asterisk reflects a statistically significant difference between values for the respective subject.
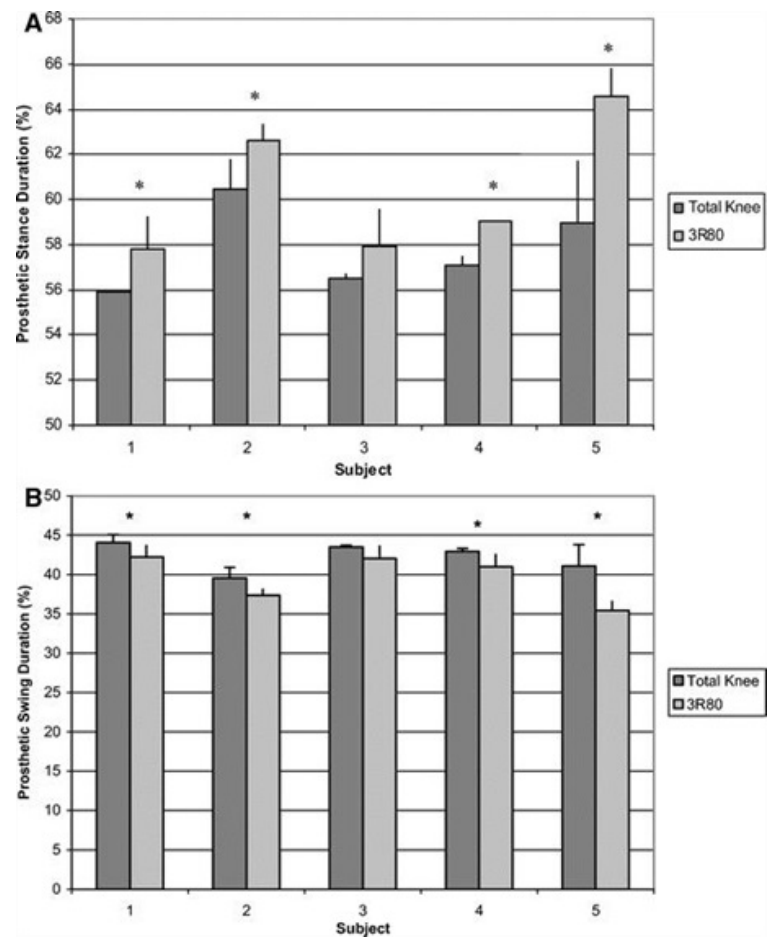

Figure 4.: Comparison of stance (A) and swing (B) duration of the prosthetic limb for all subjects when ambulating with both the Total Knee and the 3R80. The asterisk reflects a statistically significant difference between values for the respective subject. 

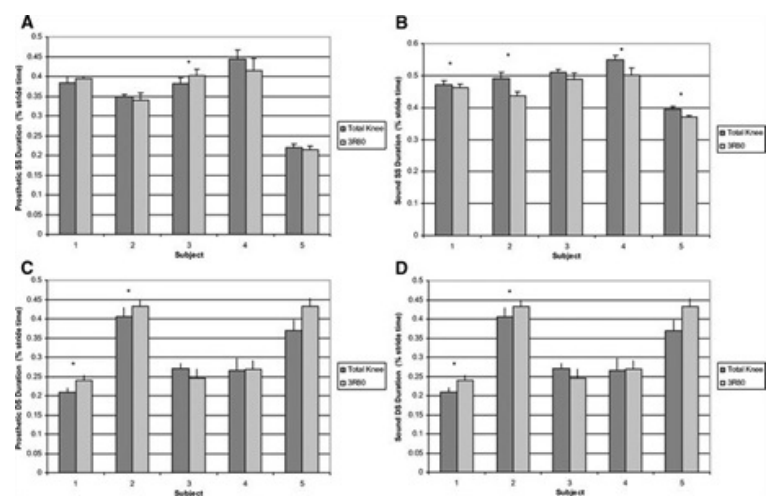

Figure 5.: Comparison of single (A: prosthetic limb, B: sound limb) and double support (C: prosthetic limb, D: sound limb) durations for each subject using the Total Knee 2000 and 3R80. The asterisk denotes a statistically significant difference between values for the respective subject.

It was expected that subjects would demonstrate the same gait pattern exhibited in TF amputees seen by Seroussi et al. ${ }^{29}$ and Schmalz et al. ${ }^{24}$ : an extension moment at the hip during loading response to maintain stability of the prosthetic knee, and a hip flexion moment in late stance to overcome the stability of the prosthetic knee to initiate swing phase. Examination of the calculated hip moments on the prosthetic side indicated that not all subjects exhibited the expected hip moment patterns, perhaps due to familiarity and comfort and/or different gait mechanisms utilized during ambulation. As such, the required hip moments based on Radcliffe's stability equation were used to ascertain which knee was more stable. Representative required hip moment data are shown in Figure $6 \mathrm{~A}$ for subject 1 ; the corresponding peak required hip extension moment during loading response ( $0 \%$ to $10 \%$ gait cycle) and peak required hip flexion moment during late-stance are shown in Figures 6B, C, respectively.
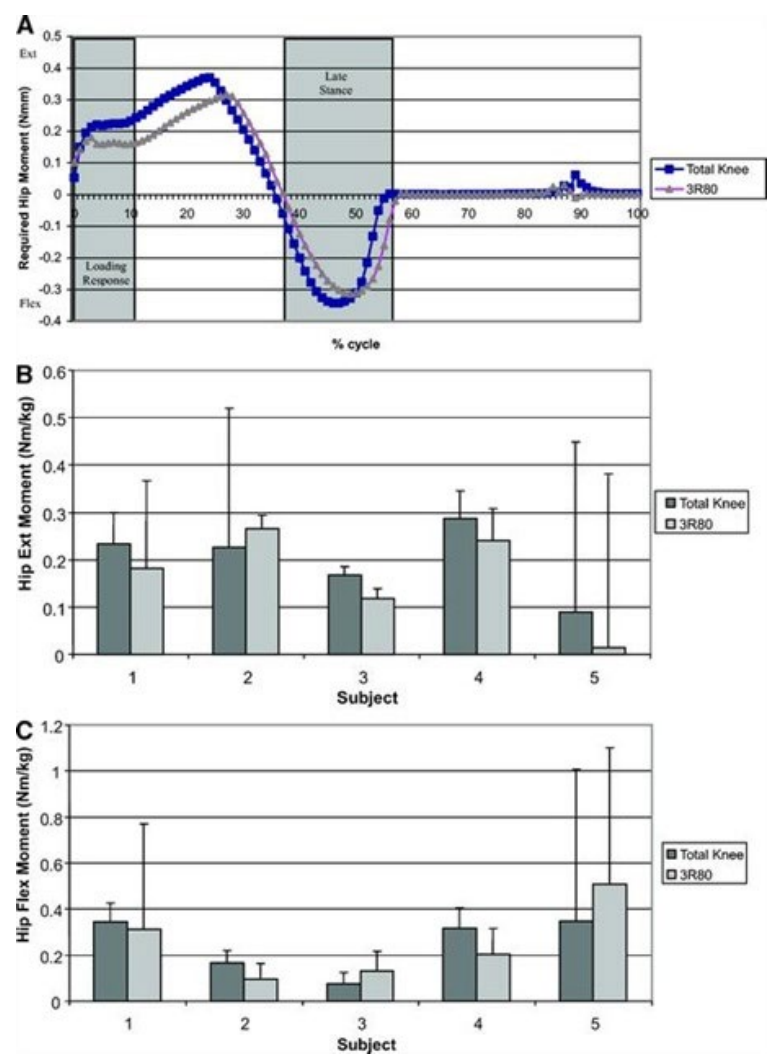

Figure 6.: Representative required hip moment for Subject 1, (A); the loading response and late stance regions are shaded. Also shown are the peak required hip extension moments during loading response (<10\% gait cycle), 
(B), and the peak required hip flexion moment during late stance/preswing (C) for each subject using the Total Knee 2000 and 3R80. None of these intra-subject differences were statistically significant.

The results of the subject questionnaires regarding the subject's perceived exertion and perception of their prosthetic limb stability with both the $3 R 80$ and Total Knee are summarized in Figure 7.
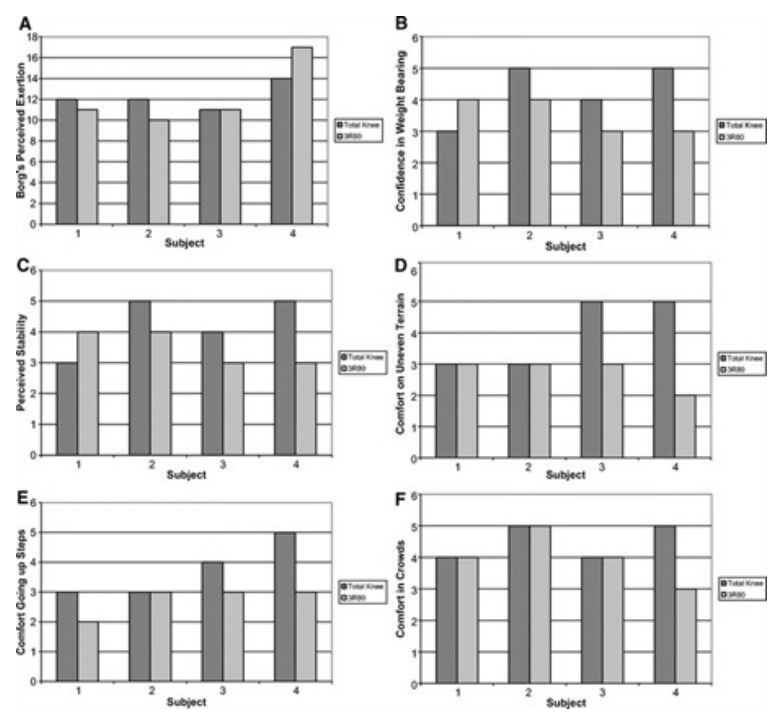

Figure 7.: Subject perceptions of exertion, stability and comfort using prostheses incorporating the $3 R 80$ and Total Knees \{lsqb;(A) perceived exertion, (B) confidence in weight-bearing, (C) perceived stability, (D) comfort on uneven terrain, (E) comfort going up steps, (F) comfort in crowds\{rsqb;.

\section{DISCUSSION}

Despite the wide variety of prosthetic knee designs available today, there remains a lack of quantitative data concerning the specific performance parameters of different types of component designs. This study used gait analysis techniques to examine the stability characteristics of two prosthetic knee component designs, the Total Knee 2000 and the $3 R 80$. The Total Knee 2000 is a polycentric prosthetic knee that utilizes a geometric lock to provide stance phase control, while the $3 R 80$ is a single axis prosthetic knee that employs a hydraulic cylinder to provide stance phase control. Both knees utilize a hydraulic mechanism for swing phase control.

During the stance phase of gait, stability of the prosthetic knee is necessary to prevent buckling of the knee. Stance phase prosthetic knee stability can be achieved both by the design and alignment of the prosthetic knee, and by the amputee exerting an extension moment at the hip. In addition to stance phase stability, prosthetic knees must also provide a smooth transition between stance and swing phases and controlled motion during swing phase. Initiation of swing typically requires the TF amputee to exert a hip flexion moment to overcome the stability of the prosthetic knee and initiate knee flexion.

The temporal and stride parameters examined in this study are closely related to one another, and their interdependence must be acknowledged when using them to evaluate stability. For example, an increase in step length may lead to longer strides, which may lead to a faster velocity if the cadence is unchanged. In addition, longer stance phase durations on one side may lead to longer steps with the contralateral limb. As a result, when considering these measures to assess prosthetic knee stability they should be interpreted as dependent measures that together may indicate increased stability, rather than independent indicators of prosthetic stability. 
Velocity, cadence, step length, stride length, stance and swing phase durations and single and double support durations were measured in this study as indicators of overall prosthetic stability. Velocity and step length in particular have been found to be correlated with the degree of lower limb impairment, with slower velocities and shorter steps indicating greater disability. ${ }^{21,30}$ Cadence is related to both velocity and step length, and subjects who take shorter steps often compensate by increasing their cadence in an attempt to maintain a consistent velocity. In TF amputees, decreased velocity and step length may be due to decreased stability on the prosthetic limb. Decreased stability on the prosthetic limb may also lead to longer sound than prosthetic stance phase durations. ${ }^{2,3}$ This increased sound limb stance phase duration typically results in longer prosthetic step lengths than sound side step lengths. Instability on a prosthetic knee may also lead to decreased prosthetic single limb support and increased prosthetic double limb support.

A prosthetic knee with increased stability should allow an amputee to walk faster and with a slower cadence due to longer step and stride lengths in comparison to a less stable knee. In addition, a more stable knee is expected to lead to longer prosthetic stance phase durations and a corresponding increase in sound limb step length, longer prosthetic single support durations, and shorter prosthetic double support durations.

The temporal and stride parameters examined in this study demonstrate that there is a difference in the stability characteristics of the Total Knee 2000 and the $3 R 80$ prosthetic knees. Figure 1A shows that all subjects walked with either a faster velocity or the same velocity with the Total Knee 2000 as compared to the 3R80, with this result being significant for 3 of the 5 subjects. Alternatively, Figure 1B shows that 4 of the 5 subjects had a lower cadence with the Total Knee 2000. A slower cadence would typically be associated with a lower velocity, unless the person was taking longer steps leading to longer strides. Figure 3 supports this conclusion by demonstrating that all subjects had longer sound side step lengths, and 4 of the 5 subjects also had longer prosthetic step length, when walking with the Total Knee 2000. Figure 2 also confirms that subjects were taking longer strides with the Total Knee 2000 with the result being significant for 4 of the 5 subjects. These measures indicate that the Total Knee 2000 is more stable than the 3R80, allowing the subjects to take longer steps and stride, thus increasing their velocity, despite a lower cadence.

Figure 4 shows the stance and swing phase durations of the gait cycle. All subjects had shorter prosthetic stance phase durations and longer prosthetic swing phase durations when walking with the Total Knee 2000. Initially, this may be interpreted to mean that the Total Knee 2000 is less stable, however, increased velocity leads to shorter stance phase durations, and 4 of the 5 subjects walked faster with the Total Knee 2000 than with the $3 R 80$.

Figure 5 displays single and double support durations for the prosthetic and sound sides. Increased prosthetic single support and decreased prosthetic double support can be interpreted as an increase in stability in the prosthetic knee. Three of four subjects demonstrated increased prosthetic single support and 4 of the 5 subjects demonstrated decreased prosthetic double support with the Total Knee 2000, once again indicating that the Total Knee 2000 seems to be more stable during stance phase than the 3 R80.

In the current study, the difference in velocity between the Total Knee 2000 and the 3R80 ranged from 0.01 to $0.07 \mathrm{~m} / \mathrm{s}$. Although these differences were large enough to be statistically significant for three subjects, they were much smaller than the differences in velocity that Chen et al. ${ }^{31}$ associated with changes in joint moments and Murray et al. ${ }^{32}$ associated with changes in muscle activity. As a result, although there were some statistically significant differences in velocity between the Total Knee 2000 and the 3R80, these differences were not likely to have influenced the hip moments calculated in this study.

Required hip moments were calculated based on the ground reaction forces and the geometry of the limb, using Radcliffe's stability equation ${ }^{14,27}$ (Figure 6 ). In examining trade-offs between stance phase stability and swing 
phase initiation effort, it was hypothesized that the knee that was more stable would require a smaller hip extension moment during loading response and a corresponding larger hip flexion moment in terminal stance. Four of the five subjects exhibited a larger required hip extension moment during loading response with the Total Knee 2000, suggesting that this knee was less stable in early stance. Based on the above hypothesis, the Total Knee 2000 should therefore have required a smaller hip flexion moment to initiate swing phase. However, the required hip flexion moment during terminal stance was less for the $3 R 80$ for subjects 1,2 , and 4 . As such, the results of this analysis showed that the relative stability characteristics of the Total Knee 2000 and the $3 R 80$ changed throughout the gait cycle. The polycentric Total Knee 2000 was shown to be more stable overall than the 3R80 based on temporal, stride parameters and stance/swing phase durations. However, the required hip moment calculations indicated that the Total Knee 2000 was less stable than the 3R80 in early stance, and that the increase in overall stability for the Total Knee 2000 was likely the result of increased mid- and late-stance stability. As a result, it was difficult to conclusively address trade-offs between stance phase stability and swing phase initiation effort using the current methodology.

As illustrated in Figure 7, subjects 1 and 4 had slightly different responses to the subjective questionnaires, likely due to the difference in residual limb length between these two subjects. Subject 1 had a long residual limb; Subject 4 had a very short residual limb. As a result, Subject 4 found the increased weight of the $3 R 80$ to be a major hindrance, as evidenced by increased sweating, need for rest between walking trials, and the subject's comments following the gait analysis session with the 3R80. This subject rated his level of exertion much higher with the 3R80 than with the Total Knee 2000, and rated the Total Knee 2000 higher in perceived stability, comfort on uneven terrain, comfort going up stairs and comfort in crowds.

There are a number of limitations that must be taken into consideration when interpreting the results of this study. Inverse dynamics is a widely used method for calculating joint moments from gait analysis data; however, the assumptions made to facilitate this method (i.e., fixed geometrical and inertial properties) introduced some error into the calculations. In addition, simplifications must be made to determine the requisite geometric and inertial parameters to input into the inverse dynamics model (i.e., estimating inertial properties from tabulated data or from a simplified geometric model; modeling the joints as hinge or ball and socket joints), and these simplifications also introduce error into the calculations. ${ }^{33,34}$

In this study, the inertial properties for the sound limb were based on tabulated data, ${ }^{33}$ while a geometric model was used to determine the inertial properties for the prosthetic limb. The geometric model was based on the model used by Miller and Childress, ${ }^{26,34}$ and was a simplification of geometric models used by other researchers to model the entire human body. ${ }^{26,35,36}$ The complexity of the human body requires complex shapes for modeling the entire human body. The lower limbs, however, can be modeled with much more simple shapes, and prosthetic components can be modeled by even simpler shapes. Despite the fact that the geometric simplifications made in this study were appropriate for the geometry of the prosthetic limbs being studied, some error was likely introduced into the calculations by assuming a uniform distribution of mass within each component of the prosthetic limb. Components such as the prosthetic pylon have uniform mass distributions; however, components such as the $3 \mathrm{R} 80$ prosthetic knee have mass concentrated in the hydraulic cylinder.

Another simplification related to the inverse dynamics model utilized in this study was the modeling of the polycentric Total Knee 2000 as a single axis knee. There is currently no standard method to track the changing instantaneous center of rotation of a joint. Motion analysis systems such as the Vicon system used in the study could be used to collect the raw marker position data for a marker set developed to track the instantaneous center of rotation of a polycentric joint; however, processing these data would require significant reprogramming of the analysis software. As a result, it is common practice to model polycentric prosthetic knees as having a single axis of rotation. ${ }^{37-40}$ 
In a case study of one TF amputee using a polycentric prosthetic knee modeled as both a single axis knee and a polycentric knee, Miller and Childress ${ }^{34}$ showed that there are only small differences in the moment at the hip during stance phase between the two models. These differences in the hip moment during stance phase were not statistically significant; however, differences in the hip moment during swing phase between the two models were found to be significant. As the current study contrasted hip moment during early (loading response) and late stance, the simplification of the polycentric knee mechanism in this study would likely not have changed the conclusions of this study.

In this study, the polycentric Total Knee 2000 was modeled as a single axis knee with the center of rotation located at the proximal anterior linkage. At initial contact, the actual knee center of rotation would have been located proximally and posterior to the knee marker. This is a more stable position for the knee center of rotation. As a result, the model used in this study may have underestimated the stability of the Total Knee 2000 in early stance. Initiation of knee flexion takes place in terminal stance with the prosthetic knee beginning in full extension. At this point, the actual knee center is still proximal and posterior to the knee marker, a more stable position.

The limited time each subject had to adjust to the prosthetic knees being investigated and their prior experience or familiarity with the types of prosthetic knees evaluated in this study may have had an effect on the results. Subjects with more experience on one of the knees may have felt more stable (i.e., biased) on that knee simply because they were more familiar with it. To try to minimize the influence of familiarity, each subject was timed walking across the gait walkway for five trials with their definitive prosthesis, and this process was repeated after gait training with the new prosthesis. The variability (e.g., standard deviation) of the subjects' velocities with each knee was compared, and as long as the variation in velocity with the new prosthesis was within $10 \%$ of that with the definitive prosthesis, they were allowed to proceed to testing. This procedure was used as an attempt to quantify the subjects' adjustment to the new prosthesis rather than relying on the subject's and the physical therapist's subjective evaluations. The results of this study indicate that the Total Knee 2000 has a higher degree of overall stability than the 3R80. As only one subject had experience with only the Total Knee 2000 , this result was not unduly biased by familiarity. Of the remaining subjects, three had experience with both types of knees and one had experience with the 3R80 only. As a result, there is no clear indication that the results of this study were skewed by familiarity with one type of prosthetic knee over the other.

The small number of subjects in this study may be perceived by some as limiting the power of the statistical analysis. However, a within subject statistical analysis was conducted in this study. In this type of analysis, the data from each subject are analyzed separately to determine if there was a significant difference between the two knees for each individual subject. This type of analysis allows for a smaller subject population while still providing sufficient power for the statistical tests, because the power of the tests is dependent, not on the number of subjects and the variation between subjects, but on the number of trials for each subject and the variation between trials for each subject. The subjects in this study displayed consistent gait with small variations between trials, resulting in sufficient statistical power (i.e., 0.7-1.00) to detect differences between the Total Knee 2000 and the 3R80 with just five walking trials with each knee. As a result, increasing the number of trials would not be recommended as the risks of fatigue greatly outweigh the small potential benefit that more trials would provide for this group of consistent ambulators. Increasing the number of subjects is recommended; however, the number of trials required would then have to be re-evaluated in light of the level of consistency exhibited by each subject participating.

\section{CONCLUSION}

The results of this study indicate that the Total Knee 2000 has a higher degree of overall stability than the 3R80; however, they also demonstrate that the relative stability characteristics of two prosthetic knees may change 
throughout the gait cycle. In this study, it was the polycentric nature of the Total Knee 2000 that resulted in differences in the alignment stability for this knee as the instantaneous center of rotation changed throughout the gait cycle. This led to differences in the relative stability characteristics of the two knees from early stance to late stance. Although the Total Knee 2000 demonstrated a higher degree of stability overall, when looking at early stance phase, the Total Knee 2000 often appeared to be less stable than the 3R80, while in late stance it appeared to be more stable.

Prosthetic knee stability is an important factor in determining which prosthetic knee component is most appropriate for a specific individual. An amputee who has particular difficulty establishing stability at heel contact may benefit from a prosthetic knee design such as the $3 R 80$, which appears to be more stable in early stance phase than the Total Knee 2000. However, an amputee who has no trouble establishing stability in early stance phase, but who encounters stability problems in mid and late stance, when their full body weight is supported on the prosthetic limb, may benefit from a prosthetic knee such as the Total Knee 2000. In addition, it is generally easier for a TF amputee to learn to establish stability early in stance phase than to correct for stability problems later in stance phase. Prosthetists and physical therapists can teach TF amputees to listen for, to feel and to see when the prosthetic knee reaches full extension to help them learn how to ensure a fully extended and stable knee for early stance phase. This suggests that it would be easier for amputees to correct for the short period of decreased stability in early stance with the Total Knee 2000 than it would be to correct for the decreased stability in mid and late stance phase with the $3 R 80$.

Although the results of this study suggest that the Total Knee 2000 performs better with respect to stability than the 3R80, there are some TF amputees for whom this knee may not be the best choice. Amputees who are healthy and very active and have good voluntary control of their prosthesis may find that the increased stability of the Total Knee 2000 limits their activity by requiring them to overcome the stability of the knee design before they can initiate swing phase. These individuals may find that the $3 R 80$ allows them to initiate swing more easily, or they may prefer a prosthetic knee that does not incorporate stance phase control, but rather relies on alignment stability and their residual limb musculature to maintain stability during stance phase. This type of knee component would be more versatile for an amputee who has the muscular ability to control the prosthesis and, as well as the endurance to maintain a high level of voluntary control for an extended period of time.

Another important factor to consider when comparing these two knee units is their varying mass. The mass of the 3R80 (approximately $1100 \mathrm{~g}$ ) is almost twice that of the Total Knee 2000 (approximately $680 \mathrm{~g}$ ). As a result, amputees with short residual limbs, such as subject 4, or amputees with muscle weakness may be unable to ambulate successfully with the $3 R 80$. In addition, the weight difference between the $3 R 80$ and the Total Knee 2000 may have biased the results of this study as subjects may have exhibited decreased stability with the $3 R 80$ due to difficulty controlling the heavier prosthesis.

In summary, the analyses conducted in this study indicated that the Total Knee 2000 had a higher degree of overall stability than the 3R80. The basis for this higher level of overall stability was the increased stability exhibited by the Total Knee 2000 in mid and late stance phases, as the Total Knee 2000 was found to be less stable than the 3R80 in early stance phase. This difference in stability between the two knees led to differences in the required hip moments between the knees, with the Total Knee 2000 requiring a larger hip extension moment during loading response due to its decreased stability at this point in the gait cycle, and a larger hip flexion moment in terminal stance due to its increased stability at this point in the gait cycle.

\section{ACKNOWLEDGMENTS}

The authors would like to thank Jason Long and the staff at the OREC Motion Analysis Laboratory at FMLH/MCW for their assistance in data collection and processing. We also acknowledge the support of Otto Bock Healthcare 
who provided the $3 R 80$ prosthetic knees, and Ossur who provided the Total Knee 2000 knee units for the study duration. The authors also acknowledge the support of David R. Del Toro, MD and Dennis Farrell, CP, who assisted with subject recruitment and assessment of clinical relevance, and Tina M. Stoeckmann, PT, PhD, who assisted with amputee gait training and study refinement.

\section{REFERENCES}

1. Hafner BJ, Sanders JE, Czerniecki J, Fergason J. Energy storage and return prostheses: does patient perception correlate with biomechanical analysis? Clin Biomech (Bristol, Avon) 2002;17:325-344.

2. Murray MP, Sepic SB, Gardner GM, Mollinger LA. Gait patterns of above-knee amputees using constantfriction knee components. Bull Prosthet Res 1980;10-34:35-45.

3. Murray MP, Mollinger LA, Sepic SB, Gardner GM, Linder MT. Gait patterns in above-knee amputee patients: hydraulic swing control vs constant-friction knee components. Arch Phys Med Rehabil 1983;64:339-345.

4. Jergesen HE, Hoaglund FT, Roberts RA, et al. The University of California Biomechanics Laboratory four-bar polycentric knee linkage. A clinical trial in 20 active above-knee amputees. Clin Orthop Relat Res 1986;184-192.

5. Czerniecki JM. Rehabilitation in limb deficiency. 1. Gait and motion analysis. Arch Phys Med Rehabil 1996;77:S3-S8.

6. Czerniecki J, Gitter AJ. Gait analysis in the amputee: has it helped the amputee or contributed to the development of improved prosthetic components?. Gait Posture 1996;4:258-268.

7. DiAngelo DJ, Winter DA, Ghista DN, Newcombe WR. Performance assessment of the Terry Fox jogging prosthesis for above-knee amputees. J Biomech 1989;22:543-558.

8. Cappozzo A, Leo T, Cortesi SS. A polycentric knee-ankle mechanism for above-knee prostheses. J Biomech 1980;13:231-239.

9. Zernicke RF, Hoy MG, Whiting WC. Ground reaction forces and center of pressure patterns in the gait of children with amputation: preliminary report. Arch Phys Med Rehabil 1985;66:736-741.

10. Romo HD. Prosthetic knees. Phys Med Rehabil Clin N Am 2000;11:595-607.

11. Michael JW. Modern prosthetic knee mechanisms. Clin Orthop Relat Res 1999;39-47.

12. Radcliffe CW. The Knud Jansen lecture: above-knee prosthetics. Prosthet Orthot Int 1977;1:146-160.

13. Oberg K. Knee mechanisms for through-knee prostheses. Prosthet Orthot Int 1983;7:107-112.

14. Radcliffe CW. Four-bar linkage prosthetic knee mechanisms: kinematics, alignment and prescription criteria. Prosthet Orthot Int 1994;18:159-173.

15. de Vries J. Conventional 4-bar linkage knee mechanisms: a strength-weakness analysis. J Rehabil Res Dev 1995;31:36-42.

16. Andrysek J, Naumann S, Cleghorn WL. Design characteristics of pediatric prosthetic knees. IEEE Trans Neural Syst Rehabil Eng 2004;12:369-378.

17. Online Technical Information 3R80. Otto Bock; 2004. Available at: http://www.ottobockus.com/ASSETS/656E10F0C4FA49D6858CD1E856B9264C/ifu 647g403 3r80FINAL.pdf.

\section{Cited Here}

18. Online Technical Information Total Knee 2000. Ossur; 2004. Available at: http://www.ossur.com/?PagelD=3490.

19. Skinner HB, Effeney DJ. Gait analysis in amputees. Am J Phys Med 1985;64:82-89.

20. Sutherland JL, Sutherland DH, Kaufman KR, Teel M. Case study forum: gait comparison of two prosthetic knee units. J Prosthet Orthot 1997;9:168.

21. Zuniga EN, Leavitt LA, Calvert JC, et al. Gait patterns in above-knee amputees. Arch Phys Med Rehabil 1972;53:373-382.

22. Macfarlane PA, Nielson DH, Shurr DG. Mechanical gait analysis of transfemoral amputees: sach foot versus the flex-foot. J Prosthet Orthot 1997;9:144.

23. Blumentritt S, Scherer HW, Michael JW, Schmalz T. Transfemoral amputees walking on a rotary hydraulic prosthetic knee mechanism: a preliminary report. J Prosthet Orthot 1998;10:61. 
24. Schmalz T, Blumentritt S, Jarasch R. Energy expenditure and biomechanical characteristics of lower limb amputee gait: the influence of prosthetic alignment and different prosthetic components. Gait Posture 2002;16:255-263.

25. Vicon Support Webpage. 2004. Available at: www.vicon.com/support.

26. Miller LA. Theories of Human Ambulation with Applications to Swing-throughCrutch Gait, (Thesis). Evanston: Biomedical Engineering, Northwestern University; 2003.

27. Boyda-Glaister CL. A Functional Comparison of Two Types of Prosthetic Knee Designs (thesis). Milwaukee: Biomedical Engineering, Marquette University; 2005.

28. Ott RL, Longnecker M. An Introduction to Statistical Methods and Data Analysis, 5th ed. Pacific Grove, CA: Thomson Learning, Inc; 2001.

29. Seroussi RE, Gitter A, Czerniecki JM, Weaver K. Mechanical work adaptations of above-knee amputee ambulation. Arch Phys Med Rehabil 1996;77:1209-1214.

30. Rose J, Gamble JG. Human Walking. Baltimore, MD: Williams and Wilkins; 1994.

31. Chen IH, Kuo KN, Andriacchi TP. The influence of walking speed on mechanical joint power during gait. Gait Posture 1997;6:171-176.

32. Murray MP, Mollinger LA, Gardner GM, Sepic SB. Kinematic and EMG patterns during slow, free, and fast walking. J Orthop Res 1984;2:272-280.

33. Yeadon MR. The simulation of aerial movement-II. A mathematical inertia model of the human body. J Biomech 1990;23:67-74.

34. Miller LA, Childress DS. Problems associated with the use of inverse dynamics in prosthetic applications: an example using a polycentric prosthetic knee. Robotica 2005;23:329-335.

35. Jensen RK, Fletcher P. Distribution of mass to the segments of elderly males and females. $J$ Biomech 1994;27:89-96.

36. McGill DJ, King WW. Engineering Mechanics: Statics and An Introduction to Dynamics. Boston, MA: PWS Publishers; 1985.

37. Boonstra AM, Fidler V, Eisma WH. Walking speed of normal subjects and amputees: aspects of validity of gait analysis. Prosthet Orthot Int 1993;17:78-82.

38. Boonstra AM, Schrama JM, Eisma WH, et al. Gait analysis of transfemoral amputee patients using prostheses with two different knee joints. Arch Phys Med Rehabil 1996;77:515-520.

39. Jaegers SM, Arendzen JH, de Jongh HJ. Prosthetic gait of unilateral transfemoral amputees: a kinematic study. Arch Phys Med Rehabil 1995;76:736-743.

40. Yokogushi K, Narita H, Uchiyama E, et al. Biomechanical and clinical evaluation of a newly designed polycentric knee of transfemoral prosthesis. J Rehabil Res Dev 2004;41:675-682.

\section{Keywords:}

transfemoral amputee gait, prostheses, prosthetic knee units 